\title{
Transabdominal ultrasonographic features in the diagnosis of gastrointestinal lymphoma
}

\author{
Maha Hasaballah ${ }^{1}$, Raafat Abdel-Malek ${ }^{2}$, Zeinab Zakaria ${ }^{1}$, Mohamad Saeed Marie ${ }^{1}$, Mohammed Sherif \\ Naguib $^{3}$ \\ ${ }^{1}$ Endemic Medicine and Hepato-gastroenterology, Faculty of Medicine, ${ }^{2}$ Clinical Oncology, Faculty of Medicine, ${ }^{3}$ Gastrointestinal Endoscopy and \\ Liver Unit, Cairo University, Cairo, Egypt \\ Contributions: (I) Conception and design: M Hasaballah; (II) Administrative support: M Hasaballah, R Abdel-Malek; (III) Provision of study materials \\ or patients: M Hasaballah, R Abdel-Malek; (IV) Collection and assembly of data: Z Zakaria, MS Marie; (V) Data analysis and interpretation: MS \\ Naguib; (VI) Manuscript writing: All authors; (VII) Final approval of manuscript: All authors. \\ Correspondence to: Zeinab Zakaria. Endemic Medicine and Hepato-gastroenterology, Faculty of Medicine, Cairo University, Cairo, Egypt. \\ Email: zenab.zakaria@yahoo.com.
}

Background: Gastrointestinal (GI) lymphoma is a challenging disease. We aimed to study and characterize the different endoscopic and transabdominal ultrasonography (TUS) features of gut lymphoma and to assess whether TUS has a complementary role to endoscopy in the diagnosis of GI lymphoma.

Methods: This study was conducted on 21 patients with GI lymphoma, attending the GI endoscopy and liver unit, Endemic Medicine Department and Oncology Department in Kasr El Aini Hospital, Cairo University. Patients were subjected to GI endoscopy (upper endoscopy \& colonoscopy) and transabdominal ultrasonography. The diagnosis was finally based on histopathology of core biopsies (obtained either endoscopically or by ultrasonography) and immuno-histochemistry.

Results: In all 21 patients with GI lymphoma included in this study, TUS could accurately determine the site of disease affection compared to endoscopy which is considered the gold standard for site localization. The main TUS pathologic features detected were increased wall thickness of the affected bowel segment with a mean value of $(15.6 \pm 5.9 \mathrm{~mm})$ and loss of layering pattern in 16 patients $(76 \%)$. While the most common endoscopic features were ulcers and mass lesions accounting for $38 \%$ of the patients for each. Diffuse large B-cell lymphoma was found in 19 patients (90\%). Because of endoscopic biopsies were conclusive in 14 patients (67\%), TUS guided biopsy was resorted to in 7 patients and was diagnostic in all of them.

Conclusions: Transabdominal ultrasonography is a useful tool in the diagnosis of GI lymphoma that is complementary to conventional diagnostic endoscopic procedures.

Keywords: Transabdominal ultrasonography (TUS); gastrointestinal lymphoma (GI lymphoma); GI endoscopy; diffuse large B-cell lymphoma (DLBCL)

Submitted Jul 10, 2018. Accepted for publication Aug 22, 2018.

doi: 10.21037/jgo.2018.09.02

View this article at: http://dx.doi.org/10.21037/jgo.2018.09.02

\section{Introduction}

Gastrointestinal tract (GIT) is rich in lymphoid tissues collectively known as gut associated lymphoid tissues (GALT) distributed in the stomach, small and large bowel. Abnormal lymphoid tissue proliferation may end up in lymphoma that may be nodal or extra nodal like in GIT.
Involvement of the gut by lymphoma may be part of systemic disease or may occur as primary disorder of the GIT which is rare (1). Stomach is commonest organ to be affected by primary lymphoma accounting for overall incidence $1-2 \%$ of all gastric malignancies however that incidence of gastric lymphoma is increasing. This may be related to wide spread of $\mathrm{H}$ pylori and higher incidence of 
mucosa-associated lymphoid tissue (MALT) lymphoma (2).

The main clinical presentations of primary GI nonHodgkin lymphoma (PGI NHL) were non-specific GI symptoms, including abdominal pain, nausea, vomiting, loss of appetite and weight loss (3). Currently, the diagnosis of PGI NHL is mainly based on endoscopic biopsies obtained from suspicious GI lesions. However, the endoscopic appearance of PGI NHL lack specificity and may vary from minimal mucosal irregularities to sizeable ulcers. Additionally, the burden of the disease is submucosal so can not be obtained by endoscopic biopsy and contributes to missed diagnosis (4).

Transabdominal ultrasonography is useful in the detection of GIT involvement in extra nodal lymphoma. Ultrasonographic features of bowel lymphoma is variable like thickened wall, echogenic center described as "bull's eye"-, "target"- or "pseudo kidney"-sign and hypoechoic (pseudo cystic) appearance of bowel wall thickening and of enlarged lymph nodes that is indicative of an intestinal lymphoma (4). It has the advantage of the panoramic view of the abdomen, the real time ability to examine the organs, bowel, and the better visualization of the bowel wall layering.

With the advances in ultrasound machines and the development of high-frequency probes, staging of GI tumors including lymphomas becomes feasible on transabdominal ultrasonography. This could be done accurately by studying the wall layering pattern and extent of invasion through the wall (4). Besides, it allows biopsy of the thickened bowel wall adds to the diagnostic power of ultrasonography.

We aimed at studying the abdominal ultrasonography and endoscopic features of GI lymphoma and to assess whether trans abdominal ultrasonography could add to the role of endoscopy in the diagnosis of GI lymphoma.

\section{Methods}

The present study was conducted on 21 patients of both sexes of an age more than 18 years from May 2014 to July 2015, primarily diagnosed as GI lymphoma attending the GI Endoscopy and Liver Unit, Endemic Medicine Department and Oncology Department in Kasr El Aini Hospital, Cairo University. All patients were recruited after a written informed consent, and this study was approved by the department ethical committee. Those previously treated or currently receiving treatment for lymphoma and with other GI malignancies were excluded from the study.

All the studied patients were subjected to: history taking, complete clinical examination, laboratory investigations, abdomino-pelvic ultrasonography, upper GI endoscopy, full colonoscopy (in patient when suspected colonic involvement) and histopathological examination of biopsy.

\section{Abdominal ultrasonography}

All patients were subjected to abdominal ultrasonographic examination including bowel examination which starts with a systemic survey using a conventional probe $(3.5-5.5 \mathrm{MHz})$ to get an overview over the abdominal organs and different parts of the GIT, subsequently detailed examination proceeds with a high-frequency probe (6-8 MHz) to obtain details of the affected bowel segments. The ultrasonographer was blinded to the endoscopic findings.

The main ultrasonographic sign of bowel disease is bowel wall thickening, (normal bowel wall thickness is 2-4 mm) (5).

\section{Endoscopy}

Patients were subjected to upper GI endoscopy and full colonoscopy (in patient when suspected colonic involvement). Lesions were characterized, biopsied and histopathologically examined. Those with negative endoscopic biopsies were subjected to rebiopsy by endoscopy, if second biopsy was negative; patients were subjected to US guided biopsies.

\section{Technique of ultrasound-guided biopsy from bowel lesions}

After localization of bowel lesions and checking good coagulation profile and platelet count, patient is sedated using titrated IV midazolam. The site of the biopsy was chosen to be shortest safest axis according to the color Doppler examination. Graded abdominal compression was applied to displace overlying normal bowel and to fix the lesion with the probe. The needle path was assessed with color Doppler sonography to ensure blood vessels would be avoided. The needle entry site on the patient's skin was marked, and the surrounding area was cleaned with betadine solution. The needle was then introduced into the thickened bowel wall avoiding transfixation of the bowel loops. An average number of four passes were made, for each procedure; while negative suction was maintained by 
Table 1 Endoscopic sites affected of the studied patients

\begin{tabular}{lc}
\hline Findings & Studied patients $(\mathrm{n}=21, \%)$ \\
\hline Site & $16(76.0)$ \\
Stomach & $5(23.8)$ \\
Duodenum & $3(14.3)$ \\
Ileum & $1(4.7)$ \\
Colorectal & \\
Sites affected & $18(85.7)$ \\
One site & $3(14.3)$ \\
More than one site &
\end{tabular}

a needle lock. After procedure was completed, the patient was observed for 4 hours to monitor his vital signs and to assess for immediate complications. The patient was given nothing by mouth for the following 6 hours and then only oral fluids were allowed for the following 24 hours.

\section{Histopathological examination}

Through endoscopic or ultrasound-guided biopsy Hematoxylin-eosin stained slides were reviewed to confirm the diagnosis. Immunohistochemical studies were performed on formalin-fixed, paraffin-embedded sections using the labeled streptavidin-biotin-peroxidase complex method with $\mathrm{DAB}$ as chromogen. The following antibodies were evaluated CD20, CD3, Kappa, lambda and cytokeratin antibodies.

\section{Statistical analysis}

All data were tabulated and statistically studied by descriptive analysis.

Comparison between groups was done using Student's $t$-test for continuous data and Chi square (2) test for categorical data. The relationship between the two diagnostic modalities was assessed using Pearson correlation coefficient (R). All tests were considered statistically significant when $\mathrm{P}<0.05$.

All statistical calculations, data management and analysis were performed using computer programs Microsoft Excel version 7 (Microsoft Corporation, NY, USA) and SPSS version 20 (Statistical Package for the Social Science; SPSS Inc., Chicago, IL, USA).

\section{Results}

A total of 21 patients with GI lymphoma were included in the present study. The median age of the studied patients was 47 years, $12(57 \%)$ patients were males and $9(43 \%)$ patients were females. Cigarette smoking was reported in (38\%) of the studied patients.

All of the patients suffered from abdominal pain, 12 patients $(57 \%)$ experienced clinically significant weight loss, 8 patients (38\%) presented with vomiting, 6 patients (28.5\%) had attacks of GIT bleeding; 5 of them in the form of haematemesis and melena while 1 patient with bleeding per rectum. Six patients (28.5\%) presented with palpable masses and 4 patients (19\%) presented with diarrhea.

Pallor, fever and lymph nodes enlargement; each of them was present in 8 patients (38\%). Lower limb edema and ascites were present each in 7 patients (33\%). Splenomegaly and hepatomegaly were recorded in (19\%) and (14\%) of the patients respectively, 3 patients (14\%) had jaundice, and 3 patients had abdominal masses (14\%).

The laboratory findings demonstrated that; the mean haemoglobin was $(10.15 \pm 2.25 \mathrm{~g})$, ESR $1^{\text {st }}$ hour was $(60 \pm 31.5 \mathrm{~mm})$, serum albumin was $(3.2 \pm 0.62 \mathrm{~g})$, LDH was $(544 \pm 223.4 \mathrm{IU})$ and Beta 2 microglobulins was $(0.282 \pm 0.008 \mathrm{mg})$.

$\mathrm{HCV}-\mathrm{Ab}$ was found to be positive in 6 patients (28\%), $\mathrm{HBsAg}$ was negative in all studied patients and $\mathrm{H}$. pylori serology was positive in 14 patients (66.6\%).

The endoscopic features of the studied patients showed that majority of our patients $(85.7 \%)$ had single site involvement. Multiple sites of involvement were found in 3 patients (14.3\%). Stomach and duodenum were involved in 2 patients while stomach, duodenum and colorectum were involved in 1 patient. The stomach was the commonest site of affection (16/21; 76\% of patients) [3 of them had other sites of affection], followed by the duodenum that was involved in $5(23.8 \%)$ patients, the ileum was affected in $3(14.3 \%)$ patients and colorectal affection was found in only 1 patient $(4.7 \%)$. The main endoscopic morphologic features were ulcers $(8 / 21 ; 38 \%)$, mass lesions $(8 / 21 ; 38 \%)$ and mucosal nodularities $(2 / 21 ; 9.5 \%)$. Thickened gastric folds were seen in 8 patients (38\%) and retained fluid was observed in 6 patients $(28.5 \%)$ (Tables 1,2).

In our study the endoscopic biopsy was positive in 9 patients (43\%) (all of them had mass lesion or an ulcer). Five patients (24\%) needed second endoscopic biopsies because of non-conclusive first biopsy. The remaining 
Table 2 Endoscopic morphologic features of the studied patients

\begin{tabular}{|c|c|c|c|c|c|c|c|c|}
\hline Site involved & \multicolumn{8}{|c|}{ Morphologic features } \\
\hline Stomach & 6 & 7 & - & 1 & 1 & 1 & 8 & 6 \\
\hline Duodenum & 2 & 1 & 1 & - & 1 & - & - & - \\
\hline Rectosigmoid & - & - & - & - & 1 & - & - & - \\
\hline Total patients No. & 8 & 8 & 2 & 1 & 1 & 1 & 8 & 6 \\
\hline$\%$ & 38 & 38 & 9.5 & 4.7 & 4.7 & 4.7 & 38 & 28.5 \\
\hline
\end{tabular}

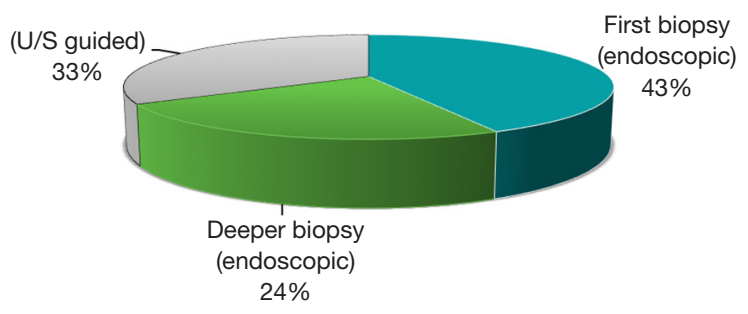

Figure 1 Percentage of endoscopic and ultrasound guided biopsies.

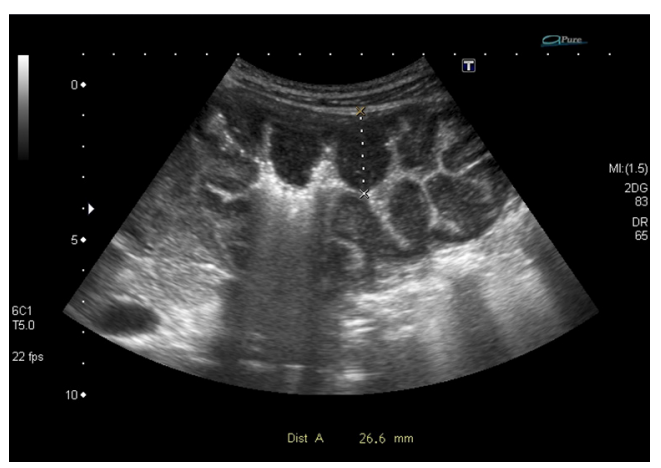

Figure 2 Thickened gastric wall.

7 patients with negative second endoscopic biopsies were subjected to trans ultrasound guided biopsy that were conclusive. No complications occurred after biopsy either endoscopic or ultrasound guided (Figure 1).

Transabdominal ultrasonographic findings of the studied patients showed that single site involvement was seen in 18 patients and multiple sites were recorded in 3 patients (stomach and duodenum involved in one patient, duodenum and jejunum in one patient and last

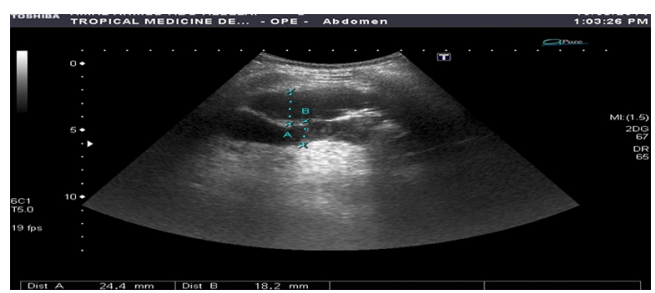

Figure 3 Thickened jejunal wall with loss of layering.

one showed wall thickening of stomach, duodenum and rectosigmoid). Fifteen patients (71.4\%) had gastric lesions, 3 patients (14.3\%) had duodenal lesions, 3 patients (14.3\%) patients had jejunal lesions, 3 patients $(14.3 \%)$ had ileal lesions. Colorectal involvement was detected in only 1 patient $(4.7 \%)$.

Bowel wall thickening $>4 \mathrm{~mm}$ is considered the most important sign of bowel disease. This detected in all patients with a mean value of $(15.6 \pm 5.9 \mathrm{~mm})$. Loss of wall layering pattern was present in $16(76 \%)$ of them (Figures 2,3).

Ultrasound guided biopsies of the bowel lesions were resorted to in 7 (33\%) patients due to non-conclusive endoscopic biopsies. All TUS guided biopsies were positive.

Comparing endoscopic morphologic features and transabdominal ultrasonographic morphologic features, we found that gastric lesions on transabdominal ultrasonography were detected in 15 patients $(71.4 \%)$ compared to 16 patients ( $76 \%$ ) by endoscopy (endoscopic submucosal bulge at gastric body in one patient proved to be jejunal thickening indenting the gastric body on transabdominal ultrasound). Duodenal wall thickening was detected in 3 patients by TUS compared to 5 patients 
Table 3 Endoscopic features compared to TUS features according to site of affection

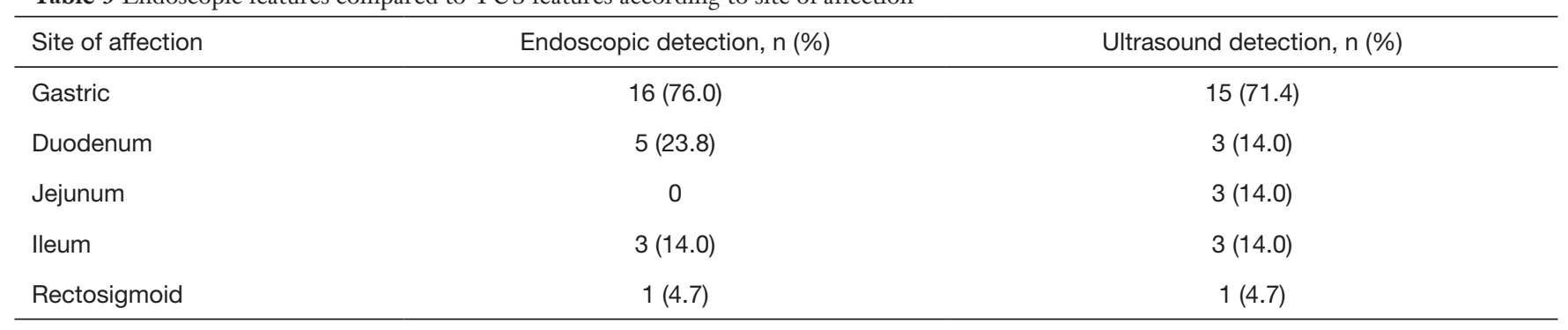

TUS, transabdominal ultrasonography.

Table 4 Correlation between endoscopic morphologic features of the lesion and the wall layering pattern by ultrasound

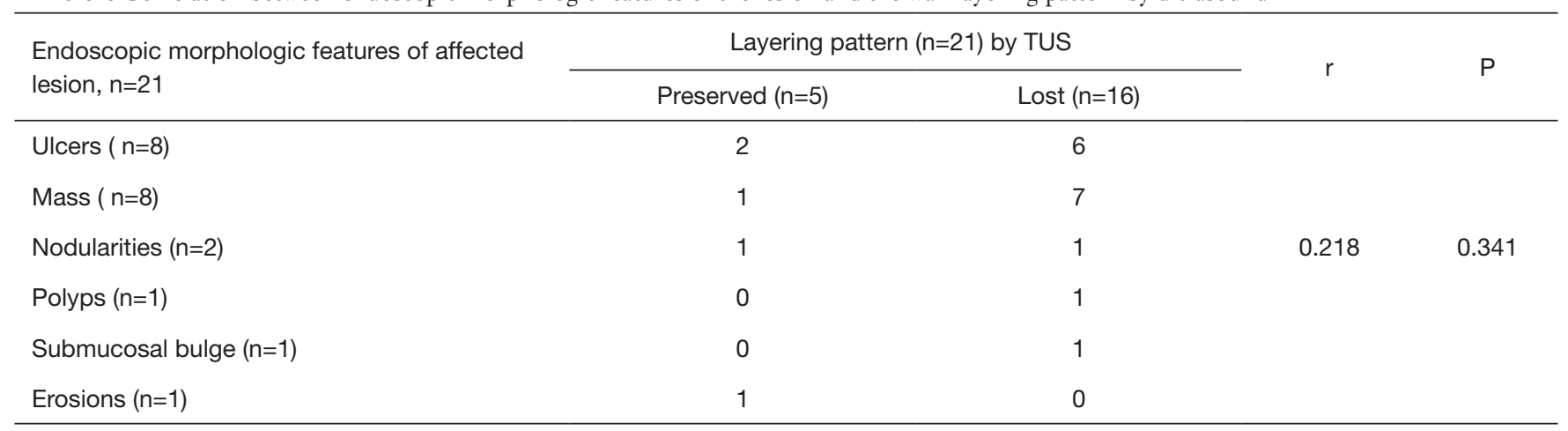

TUS, transabdominal ultrasonography.

Table 5 Correlation between endoscopy and TUS regarding retained fluid and proximal dilatation

\begin{tabular}{lccc}
\hline \multirow{2}{*}{ Endoscopic findings } & \multicolumn{2}{c}{ TUS findings } & $r$ \\
\cline { 2 - 3 } & No proximal dilatation $(\mathrm{n}=15)$ & Proximal dilatation $(\mathrm{n}=6)$ & \\
\hline No retained fluid $(\mathrm{n}=15)$ & 14 & 1 & 0.767 \\
Retained fluid $(\mathrm{n}=6)$ & 1 & 5 & $<0.001$ \\
\hline
\end{tabular}

TUS, transabdominal ultrasonography.

by endoscopy. Jejunal lesions were detected only by TUS. Three patients had ileal lesions and this was similar to that detected by colonoscopy. One patient had rectosigmoid lesion detected on colonoscopy and TUS (Table 3).

The endoscopically affected anatomical sites as (ulcers, mass) were translated as bowel wall thickening on transabdominal U/S. However, loss of wall layering pattern described on TUS did was not found on upper endoscopy $(\mathrm{r}=0.218) \mathrm{P}=0.341$ (Table 4).

Thick gastric folds on upper endoscopy were described in $8(38 \%)$ patients. This did not seem to correlate with gastric wall thickness on TUS (no statistical significance $\mathrm{P}=0.46$ ). However, the presence of retained gastric fluid on endoscopy that was described in 6 patients significantly correlated with proximal gastric dilatation on TUS $(\mathrm{P}<0.001)$ (Table 5).

The sensitivity of endoscopic biopsy in diagnosing lesions was $67 \%$ (14/21). TUS guided biopsy was resorted to in 7 patients who had inconclusive endoscopic biopsies. It was diagnostic in all of them $(7 / 7 ; 100 \%)$ from first time.

\section{Discussion}

GI tract is the most common extranodal site involved by lymphoma accounting for $5-20 \%$ of all cases (6). In the United States, gastric lymphoma is the most common 
extranodal site of lymphoma. Primary small intestinal lymphoma, accounts for up to $75 \%$ of primary GI lymphomas in the Middle East and Mediterranean basin (7).

Diagnosis of GIT lymphoma is principally a tissue diagnosis. Primary GI lymphomas are mostly of B cell lineage with very few T-cell lymphomas and Hodgkin lymphoma. Endoscopic ultrasonography is an important adjunct in staging and follow-up of gastrointestinal lymphoma (8) but it is more invasive, not readily available in all centers. Still, lesions of the small bowel and colon are out of reach to endoscopic ultrasonography.

Trans-abdominal ultrasonography is useful in the detection of GIT involvement in extra nodal lymphoma (6) being less invasive, available, comfortable to the patients and has a significant diagnostic accuracy (5).

Ghimire et al. (6) mentioned that most of the patients of GI lymphoma had an age above 50 years except for the small intestinal lymphoma that has a variable age of presentation. In our study, the median age of the studied patients was 47 years with a male predominance (57\%). This matched with other studies (9).

Most of our patients were non-cigarette smokers (62\%). In a study done by Morton et al. (10), he found that cigarette smoking seems not to affect the risk of the nonHodgkin lymphomas except for follicular lymphoma.

All of the studied patients suffered from abdominal pain $(100 \%)$, other complaints were significant weight loss, vomiting, GIT bleeding in the form of haematemesis, melena and bleeding per rectum, palpable masses and diarrhea. the same was found by Li et al. (11) and Landolsi et al. (12).

Fever (38\%), anemia (38\%), lower limb edema and ascites $(33 \%)$ were the common presenting signs in our study, while fewer patients had splenomegaly (19\%), jaundice (14\%), hepatomegaly (14\%) and abdominal masses (14\%). This agreed with Radaszkiewicz et al. (13) who stated that the physical examination is often normal, but may reveal a palpable mass and/or peripheral lymphadenopathy when the disease is advanced.

$\mathrm{HCV}$ is a known predisposing infection to lymphomas particularly lymphoplasmacytic lymphoma (Waldenstrom's macroglobulinemia) through immune complex mediated lymphoproliferation (14). In our study, $28 \%$ of our patients were positive to $\mathrm{HCV}$-antibodies. It could be explained by the high prevalence of HCV in our country (15).

Hypoalbuminemia and elevated LDH were poor prognostic parameters for GI MALT lymphoma patients (16). Beta-2 microglobulin is a potent prognostic marker in malignant lymphomas $(17,18)$.

In this study, the overall laboratory findings of the studied patients, particularly $\mathrm{Hb} \%$, serum albumin, $\mathrm{LDH}$ and beta 2 microglobulins were in different levels than the normal value of each.

Lim et al. (19) found an association between HBV infection and non-Hodgkin lymphoma. However, HBV infection does not appear to have a significant impact on the clinical presentations and prognosis of NHL. In our study all patients had negative HBAg.

Chronic infection by $\mathrm{H}$. pylori is a stimulus to a chronic immune-inflammation that is induced in mucosal barriers as part of a normal adaptive immune response (acquired mucosa associated lymphoid tissue MALT) (20). The best evidence of the etiologic link for the association between Helicobacter pylori-positive gastritis and gastric MALT lymphoma, is that the successful eradication of $\mathrm{H}$. pylori with antibiotics can be followed by gastric MALT lymphoma regression in more than $2 / 3$ of cases (21). In our study 14/21 (66.6\%) were positive for H. pylori which was higher than that reported by Song et al. [2013], 51.4\% of their cases and Feng et al. [2014], 34\% (22,23).

In the present study stomach was the commonest site of affection (76\%) detected by endoscopy and ultrasound, followed by the duodenum (23.8\%), the ileum (14.3\%) and the rectosigmoid $(4.7 \%)$ while the jejunum was not included as we did not use an enteroscope. Multiple sites of GIT involvement were detected in 3 patients (14.3\%). In a large study included 101 patients with non-Hodgkin primary gut lymphoma, stomach affection accounted for $58.4 \%$, intestinal affection in $39.6 \%$, while multiple GI involvements was reported in $1.98 \%$ (22).

We described the endoscopic features of gastrointestinal lymphoma mainly were ulcers and mass lesion accounting for $38 \%$ for each of them. Other features including mucosal nodularities, erosions, polyps and submucosal bulge detected but in fewer numbers of patients. The same findings were observed by Nonaka et al. (24) who mentioned that findings of gastric lymphoma are variable including superficial ulcer, erosion, gastritis, discolored or cobblestone areas, early cancer-like mass and mixed types.

The main ultrasonographic features detected by transabdominal ultrasound in our patients were increased wall thickness (seen in all patients with a mean value of $(15.6 \mathrm{~mm})$ in relation to the normal median value of each site $(\sim 5$ folds), loss of layering pattern present in 16 patients $(76 \%)$, other features included hypoechogenicity of the wall, the proximal dilatation, and the perilesional 
lymph gland enlargement. Ultrasound can be used as tool for characterization and differentiation between benign and malignant bowel lesions based on the degree of wall thickness, loss of wall layering and length of affected segment, however other indices like resistivity index (RI), pulsatility index (PI) of the intramural vessels and superior mesenteric artery (SMA) showed no difference between the inflammatory and malignant groups (25).

Endoscopy provided $67 \%$ positivity for diagnosis; first endoscopic biopsy was positive in $43 \%$ (9/21) of patients while a second deeper endoscopic biopsy was required in 5 patients (24\%). Transabdominal ultrasonographic guided biopsy was resorted to in 7 patients (33\%) to confirm diagnosis that was positive in all patients from first time. This may be related to the submucosal nature of the tumor. TUS guided biopsy provides larger size of biopsy $(\sim 1 \mathrm{~cm}$ core) in comparison to the endoscopic biopsy (few $\mathrm{mm}$ core). More importantly TUS guided biopsy allows full thickness biopsy of the bowel wall (from the serosa down to the mucosa). Ouakaa-Kchaou et al. (26) reported that under endoscopic examination the lesions were mainly identified as ulcers and ulcerations (93.75\%) and the endoscopic biopsy confirmation rate reached $87.5 \%$ when biopsies were repeated.

TUS guided biopsies gave a specific diagnosis in GIT lesions in $95.2 \%$ (40/42) of cases in a study done by MarcoDoménech et al. [2001] (27). Ultrasound-guided bowel wall core biopsy had sensitivity of $98.2 \%$ in diagnosing malignant lesions and specificity of $66.6 \%$ in benign lesions (28).

\section{Conclusions}

Transabdominal ultrasonography is still a useful tool in the diagnosis of gastrointestinal lymphoma and is complementary to conventional diagnostic endoscopic procedures.

\section{Acknowledgements}

None.

\section{Footnote}

Conflicts of Interest: The authors have no conflicts of interest to declare.

Ethical Statement: This study was approved by the local ethical committee of Clinical Oncology Department in Kasr El Aini Hospital, Cairo University and his committee meets monthly to approve clinical research and our research registered in April 2014. Informed consent was taken from all patients.

\section{References}

1. Shenkier TN, Connors JM. Primary extranodal nonHodgkin's lymphomas. In: Canellos G, Lister TA, Young B, editors. The Lymphomas. 2nd edition. Philadelphia, PA: Saunders Elsevier 2006;325-47.

2. Andrews CN, John Gill M, Urbanski SJ, et al. Changing epidemiology and risk factors for gastrointestinal non-Hodgkin's lymphoma in a North American population: population-based study. Am J Gastroenterol 2008;103:1762-9.

3. Koch P, del Valle F, Berdel WE, et al. Primary gastrointestinal non-Hodgkin's lymphoma: I. Anatomic and histologic distribution, clinical features, and survival data of 371 patients registered in the German Multicenter Study GIT NHL 01/92. J Clin Oncol 2001;19:3861-73.

4. Hollerweger A, Dirks K, Szopinski K. Transabdominal ultrasound of the gastrointestinal tract. Available online: http://www.kosmos-host.co.uk/efsumb-ecb/coursebooktransgit_ch08.pdf

5. Roccarina D, Garcovich M, Ainora ME, et al. Diagnosis of bowel diseases: The role of imaging and ultrasonography. World J Gastroenterol 2013;19:2144-53.

6. Ghimire P, Wu GY, Zhu L. Primary gastrointestinal lymphoma. World J Gastroenterol 2011;17:697-707.

7. Freedman AS. Clinical presentation and diagnosis of primary gastrointestinal lymphomas. Available online: https://www.uptodate.com/contents/clinical-presentationand-diagnosis-of-primary-gastrointestinal-lymphomas

8. Bautista-Quach MA, Ake CD, Chen M, et al. Gastrointestinal lymphomas: Morphology, immunophenotype and molecular features. J Gastrointest Oncol 2012;3:209-25.

9. Aledavood A, Nasiri MR, Memar B, et al. Primary gastrointestinal lymphoma. J Res Med Sci 2012;17:487-90.

10. Morton LM, Hartge P, Holford TR, et al. Cigarette smoking and risk of non-Hodgkin lymphoma: a pooled analysis from the International Lymphoma Epidemiology Consortium (interlymph). Cancer Epidemiol Biomarkers Prev 2005;14:925-33.

11. Li M, Zhang L, Wu N, et al. Imaging findings of primary splenic lymphoma:a review of 17 cases in which diagnosis 
was made at splenectomy. PLoS One 2013;8:e80264.

12. Landolsi A, Chabchoub I, Limem S, et al. Primary digestive tract lymphoma in central region of Tunisia: anatomoclinical study and therapeutic results about 153 cases. Bull Cancer 2010;97:435-43.

13. Radaszkiewicz T, Dragosics B, Bauer P. Gastrointestinal malignant lymphomas of the mucosa-associated lymphoid tissue: factors relevant to prognosis. Gastroenterology 1992;102:1628-38.

14. Foster LH, Portell CA. The role of infectious agents, antibiotics, and antiviral therapy in the treatment of extranodal marginal zonelymphoma and other low-grade lymphomas. Curr Treat Options Oncol 2015;16:28.

15. El-Zanaty F, Way A. Egypt Demographic and Health Survey 2008. Cairo. Ministry of Health, El-Zanaty and Associates 2009.

16. Virijevic M, Perunicic-Jovanovic M, Djunic I, et al. Pretreatment risk factors for overall survival in patients with gastrointestinal and non-gastrointestinal mucosa associated lymphoid tissue lymphomas. J BUON 2014;19:178-82.

17. Yao K. The endoscopic diagnosis of early gastric cancer. Ann Gastroenterol 2013;26:11-22.

18. Miyashita K, Tomita N, Taguri M, et al. Beta-2 microglobulin is a strong prognostic factor in patients with DLBCL receiving R-CHOP therapy. Leuk Res 2015. [Epub ahead of print].

19. Lim ST, Fei G, Quek R, et al. The relationship of hepatitis $B$ virus infection and non-Hodgkin's lymphoma and its impact on clinical characteristics and prognosis. Eur J Haematol 2007;79:132-7.

20. Pereira MI, Medeiros JA. Role of Helicobacter pylori in gastric mucosa-associated lymphoid tissue lymphomas.

Cite this article as: Hasaballah M, Abdel-Malek R, Zakaria Z, Marie MS, Naguib MS. Transabdominal ultrasonographic features in the diagnosis of gastrointestinal lymphoma. J Gastrointest Oncol 2018;9(6):1190-1197. doi: 10.21037/ jgo.2018.09.02
World J Gastroenterol 2014;20:684-98.

21. Vannata B, Stathis A, Zucca E. Management of the marginal zone lymphomas. Cancer Treat Res 2015;165:227-49.

22. Song LN, Cen XN, Ou JP, et al. Clinical and prognostic analysis of 101 cases of primary gastrointestinal nonHodgkin's lymphoma. Zhongguo Shi Yan Xue Ye Xue Za Zhi 2013;21:387-91.

23. Feng J, Wan W, Wan W, et al. Clinicopathological characteristics and prognostic analysis of 92 cases with primary gastrointestinal diffuse large B-cell lymphoma. Zhonghua Xue Ye Xue Za Zhi 2014;35:288-94.

24. Nonaka K, Ohata K, Matsuhashi N. Endoscopic Diagnosis of Gastric MALT Lymphoma. J Cancer Ther 2014;5:448-52.

25. Hasab Allah MS, Al-Kady NM, El Etreby RM. Differentiation of benign and malignant bowel lesions by transabdominal ultrasound using grey-scale and Doppler parameters. Arab J Gastroenterol 2014;15:148-56.

26. Ouakaa-Kchaou A, Gargouri D, Kochlef A, et al. Clinicopathologic characteristics of low grade primary gastric non-Hodgkin's lymphomas: experience from a single center. Tunis Med 2011;89:676-81.

27. Marco-Doménech SF, Gil-Sánchez S, Fernández-García $\mathrm{P}$, et al. Sonographically guided percutaneous biopsy of gastrointestinal tract lesion. AJR Am J Roentgenol 2001;176:147-51.

28. Hasab Allah MS, El-Hindawy AA. Percutaneous ultrasound-guided bowel wall core biopsy: a nonconventional way of diagnosis of gastrointestinal lesions. Surg Endosc 2013;27:3187-96. 